\title{
HUMANITIES \\ The challenge of breaking bad news
}

\author{
Sana Basseri BSc PhD', David Haase MBBS FRCPC 2 \\ ${ }^{1}$ Class of 2018, Faculty of Medicine, Dalhousie University \\ ${ }^{2}$ Division of General Internal Medicine, Department of Medicine, Dalhousie University
}

Y

ou are called to the Emergency Department to assess a 63-year-old man with acute onset of shortness of breath. He has no known health conditions but has a 40 pack-year smoking history. He thinks that he may have the flu since his wife was just recovering from flu-like symptoms. He has not seen a healthcare provider in quite some time and this is his first visit to the hospital. Following some initial workup and imaging which showed a collapsed right lung, a chest CT scan was ordered which revealed metastatic lung cancer. As the physician, how would you approach informing the patient of his diagnosis?

Bad news can be defined as any information that can drastically and negatively change a person's expectations or views about their future. ${ }^{1}$ While typical examples of bad news in the medical context include the diagnosis of terminal illness, it is important to step back and consider a wide spectrum of physical, emotional, social, and occupational factors that may impact a patient and thus could be considered bad news for that individual or their family. ${ }^{1}$

Breaking bad news is a difficult and complex communication skill to acquire yet one that is essential for physicians. How bad news is delivered can have tremendous implications not just for patients and their families, but also for the physician. Developing this communication skill requires practice, self-reflection, and flexibility to adapt one's approach according to a given situation as well as to patient preferences, behavior, and understanding. While the focus of this article is on physicians, we acknowledge that other health care professionals are also frequently involved in such discussions and hence may also benefit from this article. $^{2}$

\section{Challenges with breaking bad news}

Physicians frequently encounter situations requiring communication of bad news to patients and their families; however, doing so effectively and in a sensitive and empathetic manner still remains one of the most challenging tasks in medicine. When faced with a situation or illness that has no remedies, physicians can feel powerless and may fear extinguishing a patient's hope. Physicians may also feel guilty or experience a sense of failure, and are often without adequate preparation to deal with their own emotions. ${ }^{1}$ Patients receiving bad news could express a range of emotions such as shock, anger, fear, sadness or denial, which require a physician's attention, empathy and appropriate response. ${ }^{3}$ Physicians may feel inadequate, lack sufficient skill, or be reluctant to deal with the patient's emotional response. Furthermore, as bearers of bad news, physicians may want to avoid altering the patient's perception of their doctor and may fear being viewed more negatively.

A recent randomized controlled trial sought to compare patients' perception of physician compassion by having them watch videos depicting delivery of a more optimistic versus a less optimistic message, while keeping body language and use of empathetic statements identical. ${ }^{4}$ Interestingly, but perhaps not surprisingly, patients perceived greater compassion and trustworthiness in the physician conveying the optimistic message. ${ }^{4}$ The findings of this study suggest that it is the actual content of the message, independent of factors such as communication skills and empathy, that may have an impact on how patients perceive their physician.

Physicians are tasked with the delicate role of simultaneously fostering optimism and realism (i.e. ensuring that patients do not lose all sense of hope while also communicating realistic expectations). ${ }^{3}$ It is only recently that medical schools have started to integrate communication skills into the curriculum, including strategies for breaking bad news or discussing medical errors. Still, physicians face many barriers to effectively and empathetically communicate bad news. Often, there may be insufficient time for building a good rapport and developing a therapeutic relationship with the patient prior to the delivery of bad news. Additionally, a hectic clinical practice or hospital environment can leave the physician with little choice regarding the setting or time at which these conversations take place, particularly when there are competing responsibilities that require the physician's attention. ${ }^{5,6}$ Despite these challenges, supporting patients in their time of need by providing a therapeutic presence can be incredibly rewarding both personally and professionally.

\section{Impact on patients}

Evidence suggests that the way in which news is delivered can to some extent worsen or alleviate the patient's distress and anxiety. ${ }^{8}$ Factors that patients perceive to contribute to poor delivery of bad news 
include excessive bluntness, inappropriate location or time for serious discussion, and failure to maintain hope. ${ }^{3}$ Poor communication of bad news can result in feelings of mistrust, anger, fear and blame, and can lead to adverse outcomes or unnecessary suffering. ${ }^{9}$ In cancer patients for instance, poor communication is associated with worse clinical and psychosocial outcomes, including worse pain control, reduced adherence to treatment, confusion over prognosis, and dissatisfaction. ${ }^{10}$ On the other hand, studies show that use of honest and unambiguous language allows cancer patients to develop better coping skills. ${ }^{8}$

In an article describing the patient's perspective on breaking bad news, one cancer patient who had multiple surgical procedures recalls the positive impact of finding someone who was able to 'lift her spirits' and make her feel as part of the team. On the other hand, she also expressed fear of one day being abandoned by her oncologist should all treatments fail. ${ }^{11}$ These are not infrequent experiences faced by sick patients, and so it is clear that along with good communication skills and patient centered care, physicians must also acknowledge patient fears and concerns. A balance between hoping for the best and preparing for the worst allows patients to get their personal affairs in order and dedicate time to what they find most valuable or meaningful in life. Even when no curative options are available, palliative care and an open line of communication can have a powerful therapeutic impact on a patient's life.

\section{Impact on physicians}

For the clinician, communication difficulties can lead to poor job satisfaction and high levels of stress, as well as increased risk of medical errors and/or complaints. On the other hand, improved communication between doctors and patients improves compliance, and reduces the incidence of litigation. ${ }^{12-14}$ Most doctors rate the task of breaking bad news as stressful and report experiencing anticipatory stress responses as well as feelings of dread, nervousness, and anxiousness. ${ }^{6,15}$ However, relatively few studies have empirically examined the effect of breaking bad news on physicians. A recent qualitative study explored the range of cognitive appraisal and coping responses used by physicians in relation to breaking bad news. ${ }^{15}$ Based on a revised transactional model of stress and coping, physicians' approach was categorized as either 1) problem-focused coping (e.g. changing or controlling the situation), 2) emotion-focused coping (e.g. managing negative emotional responses) or 3) meaning-focused coping (e.g. finding benefit or underlying meaning in the negative experience). In comparison to junior doctors, senior doctors were more likely to use prob- lem-focused coping (which also included limiting exposure or distancing themselves from breaking bad news scenarios), as well as meaning-focused coping strategies such as cognitively reframing the stressful situation in a positive way. ${ }^{15}$ These findings suggest that we need a better understanding of the impact of delivering bad news on physicians in order to increase physician awareness and improve training on coping strategies. It is important for physicians to seek support through formal or informal debriefing, and consult with colleagues and seek advice when in doubt. ${ }^{7}$

\section{How should bad news be delivered?}

Communicating bad news to patients has several important characteristics. These include: the physician's attitude, the clarity of the message, the ability to answer questions, and attention to privacy. ${ }^{5}$ Ideally, the person delivering bad news will have a therapeutic relationship with the patient, or be someone with experience and skill. However, at times the situation may be less than ideal, and hence it becomes important for all physicians to be prepared with an effective and empathetic approach to such conversations.

Table 1 outlines a number of strategies that have been developed around the world for delivering bad news. The reader is encouraged to access the original papers referenced in the table for full descriptions. While some strategies such as the SPIKES protocol have been widely accepted and taught, recent studies suggest that such approaches for breaking bad news must be evaluated in the context of patient preferences. ${ }^{16,17}$ As the authors of the SPIKES protocol suggest, it is meant to be a flexible approach to account for differences in the patient population. ${ }^{16}$ For instance, a recent study conducted in Germany aimed at evaluating patient satisfaction with approaches to delivering bad news concluded that the SPIKES protocol could benefit from some modifications. ${ }^{17}$ These include reassurance of listeners' understanding by using a 'ask-tell-invite' approach, respect for prearrangement needs, and bad-news delivery in a two-step procedure. ${ }^{17}$ About $50 \%$ of patients surveyed desired a second conversation preferably with a relative or loved one present, suggesting that this is an important step to implement when delivering bad news. ${ }^{17}$

Finally, attention must also be given to cultural diversity and language barriers which may benefit from having a culturally specific support person or interpreter present. ${ }^{7}$ Appropriate spiritual support should also be considered. Hence, while consensus guidelines on breaking bad news are helpful for clinicians to evaluate and improve their communication skills, their clinical efficacy needs to be further established to ensure the 
Table I. Examples of guidelines, strategies or mnemonics for breaking bad news

\begin{tabular}{|c|c|}
\hline Study/Author & Guideline / Protocol / Mnemonic \\
\hline $\begin{array}{l}\text { Girgis A et al. } 1998 \\
\text { Australia }^{8}\end{array}$ & $\begin{array}{l}\text { Steps: Ensure privacy and adequate time, assess understanding, provide } \\
\text { information simply and encourage patients to express feelings, give broad } \\
\text { time frame, arrange review, discuss treatment options, offer assistance to tell } \\
\text { others, provide information about support services, document information } \\
\text { given }\end{array}$ \\
\hline $\begin{array}{l}\text { SPIKES } \\
\text { Baile WF et al. } 2000 \\
\text { USA }{ }^{16}\end{array}$ & $\begin{array}{l}\text { S: SETTING up the interview, } \\
\text { P:Assessing the patient's PERCEPTION, } \\
\text { I: Obtaining the patient's INVITATION, } \\
\text { K: Giving KNOWLEDGE and information to the patient, } \\
\text { E:Addressing the patient's EMOTIONS with empathetic responses, } \\
\text { S: STRATEGY and SUMMARY }\end{array}$ \\
\hline $\begin{array}{l}\text { ABCDE } \\
\text { VandeKieft GK. 200I } \\
\text { USA }^{5}\end{array}$ & $\begin{array}{l}\text { A:Advance preparation } \\
\text { B: Build a therapeutic environment/relationship } \\
\text { C: Communicate well } \\
\text { D: Deal with patient and family reactions } \\
\text { E: Encourage and validate emotions }\end{array}$ \\
\hline $\begin{array}{l}\text { BREAKS } \\
\text { Narayanan } \mathrm{V} \text { et al. } 2010 \\
\text { India }^{9}\end{array}$ & $\begin{array}{l}\text { Background: patient's disease status, emotional status, coping skills, educa- } \\
\text { tional level, and support system available } \\
\text { Rapport } \\
\text { Explore: start from what the patient knows about his/her illness and then } \\
\text { what he/she thinks about the disease/diagnosis } \\
\text { Announce: a warning shot is desirable, get consent and then announce diag- } \\
\text { nosis without use of medical jargon } \\
\text { Kindle: ask the patient to recount what they have understood while trying } \\
\text { to avoid stating any unrealistic treatment options } \\
\text { Summarize: go over main points and patient's concerns, patient should be } \\
\text { assured of physician's active participation in ongoing care plans }\end{array}$ \\
\hline $\begin{array}{l}\text { A SIX-STEP APPROACH } \\
\text { Old JL. } 201 \text { I } \\
\text { USA }^{7}\end{array}$ & $\begin{array}{l}\text { I. Assess the patient's understanding: "What do you know about your con- } \\
\text { dition?" or "What have the doctors told you?" } \\
\text { 2. Give a "warning shot": "I'm sorry, I have bad news." } \\
\text { 3. Present the bad news using words the patient will understand. } \\
\text { 4. Be quiet and listen. } \\
\text { 5. Give additional information in layers as requested by the patient or family. } \\
\text { 6. Follow up:This is the beginning of a journey with the patient. }\end{array}$ \\
\hline
\end{tabular}

guidelines are evidence-based and in line with patient preferences.

\section{Suggestions for avoiding pitfalls}

- Prepare by reading the chart notes and obtain the appropriate information before starting a conversation with the patient

- Select a private setting and involve family members if the patient prefers

- Minimize disruptions and avoid non-verbal cues which may suggest lack of time

- Include appropriate pauses to ensure information is understood, the patient has a chance to express him/ herself, and ask questions before continuing

- If possible, have a nurse or other member of patient's care team present so that the patient and their family can speak to someone after the physician leaves

- Warn the patient of what's to come, for example "I'm sorry, I have bad news." ${ }^{7}$

- Avoid use of medical jargon, for example, say 'spread' instead of 'metastasize' 16

- Look for cues that may suggest the patient is not coping well and be prepared to end the consultation

- Do not attempt to deliver all the information at once, plan for a second meeting

- Avoid euphemisms. For example, instead of "we recommend a switch to comfort care," say: "Treatments are failing or have failed, but comfort will of course be maintained" 18

- Avoid phrases that will cause the patient to lose all hope, such as "there's nothing more we can do for you", but at the same time be careful to avoid false hope or unrealistic expectations which can mislead the patient 
and their family

- Validate the patient's feelings, but avoid saying "I know how you feel," unless you really do. Instead, you could say "I know this wasn't what you wanted to hear. I wish I had better news," ${ }^{16}$ or "I'm sorry," even "I don't know what to say" can be beneficial ${ }^{7}$

- If asked "How long have I got to live?" it is best to provide a broad, realistic time frame and avoid giving a definite time scale ${ }^{8}$

- Do not criticize a colleague's medical care as this will likely not be helpful

- Do not ignore your own needs whether for support or advice on a patient's situation

\section{Future directions}

A growing body of evidence demonstrates that both patients and physicians would benefit if physicians gained more training and confidence in delivering bad news. Today, an increasing number of medical students and residents have received some level of exposure to communication skills and strategies for breaking bad news through their medical education. However, to be effective, providing experiential training in the actual behavioural skills is likely more beneficial than providing the guidelines alone. A Canadian qualitative study of first and second-year medical and surgical residents conducted over a decade ago, found that despite an ability to identify several important guidelines for breaking bad news, many did not practice them due to a number of barriers. ${ }^{19}$ These included lack of support from other healthcare professionals, time constraints, personal fears, and lack of opportunities for discussion or adequate supervision. ${ }^{19}$ Protocols such as SPIKES, are frameworks which should be guided by each individual situation and patient, hence requiring the physician to be flexible in his or her approach.

Remarkably, studies suggest that while training can boost confidence and effectiveness in delivering bad news, such training does not necessarily reduce the stress involved in delivering bad news. ${ }^{6}$ Research into strategies for structuring less optimistic messages to still convey a physician's compassion may be beneficial in reducing the burden faced by physicians delivering bad news. ${ }^{4}$ Moreover, with physician mental health and well-being in mind, educational efforts should also focus on enhancing coping responses and stress management in future healthcare professionals.

\section{Conclusions}

Good communication skills are critically important during delivery of difficult or bad news to patients and their families. Breaking bad news in the healthcare setting is often quite challenging, but it is an essential skill for physicians. Given the significant impact that delivery of bad news has on patients, their families, and the healthcare provider, strategies for breaking bad news is an area that has generated significant research. A variety of suggested approaches have been proposed and utilized in medical education. Increased awareness and early training during medical school and residency is an important step towards gaining adequate skill, experience, and confidence. Developing an effective and empathetic approach would not only enhance patient care and be beneficial to patients and their families, but may also reduce the stress and emotional burden on physicians delivering bad news.

\section{References}

1. Fallowfield L, Jenkins V. Communicating sad, bad, and difficult news in medicine. Lancet 2004;363(9405):312-9.

2. Baer L, Weinstein E. Improving oncology nurses' communication skills for difficult conversations. Clin J Oncol Nurs 2013;17(3):4551.

3. Back AL, Arnold RM, Quill TE. Hope for the best, and prepare for the worst. Ann Intern Med 2003;138(5):439-43.

4. Tanco K, Rhondali W, Perez-Cruz P, Tanzi S, Chisholm GB, Baile W, et al. Patient perception of physician compassion after a more optimistic vs a less optimistic message: a randomized clinical trial. JAMA Oncol 2015;1(2):176-83.

5. VandeKieft GK. Breaking bad news. Am Fam Physician 2001;64(12):1975-8.

6. Ptacek JT, McIntosh EG. Physician challenges in communicating bad news. J Behav Med 2009;32(4):380-7.

7. Old J. Communicating bad news to your patients. Fam Pract Manag 2011;18(6):31-5.

8. Girgis A, Sanson-Fisher RW. Breaking bad news. 1: Current best advice for clinicians. Behav Med 1998;24(2):53-9.

9. Narayanan V, Bista B, Koshy C. 'BREAKS' Protocol for Breaking Bad News. Indian J Palliat Care 2010;16(2):61-5.

10. Hanratty B, Lowson E, Holmes L, Grande G, Jacoby A, Payne S, et al. Breaking bad news sensitively: what is important to patients in their last year of life? BMJ Support Palliat Care 2012;2(1):24-8.

11. Dias L, Chabner BA, Lynch TJ, Jr., Penson RT. Breaking bad news: a patient's perspective. Oncologist 2003;8(6):587-96.

12. Zolnierek KB, Dimatteo MR. Physician communication and patient adherence to treatment: a meta-analysis. Med Care 2009;47(8):826-34.

13. Levinson W, Roter DL, Mullooly JP, Dull VT, Frankel RM. Physician-patient communication. The relationship with malpractice claims among primary care physicians and surgeons. JAMA 1997;277(7):553-9.

14. Eastaugh SR. Reducing litigation costs through better patient communication. Physician Exec 2004;30(3):36-8.

15. Shaw JM, Brown RF, Dunn SM. A qualitative study of stress and coping responses in doctors breaking bad news. Patient Educ Couns 2013;91(2):243-8.

16. Baile WF, Buckman R, Lenzi R, Glober G, Beale EA, Kudelka AP. SPIKES-A six-step protocol for delivering bad news: application to the patient with cancer. Oncologist 2000;5(4):302-11.

17. Seifart C, Hofmann M, Bar T, Riera Knorrenschild J, Seifart U, Rief W. Breaking bad news-what patients want and what they get: evaluating the SPIKES protocol in Germany. An Oncol 2014;25(3):707-11.

18. Workman SR. Never say die?--as treatments fail doctors' words must not. Int J Clin Pract 2011;65(2):117-9.

19. Dosanjh S, Barnes J, Bhandari M. Barriers to breaking bad news among medical and surgical residents. Med Educ 2001;35(3):197205. 